\title{
Article \\ An Observational Cohort Study on 194 Supraglottic Cancer Patients: Implications for Laser Surgery and Adjuvant Treatment
}

\author{
Gerhard Dyckhoff ${ }^{1, *(\mathbb{D}}$, Rolf Warta ${ }^{1}{ }^{1}$, Christel Herold-Mende $\left.{ }^{1}{ }^{(}\right)$, Elisabeth Rudolph ${ }^{2}$, Peter K. Plinkert ${ }^{1}$ \\ and Heribert Ramroth ${ }^{2}$
}

1 Department of Otorhinolaryngology, Head and Neck Surgery, University of Heidelberg, 69120 Heidelberg, Germany; Christel.Herold-Mende@med.uni-heidelberg.de (C.H.-M.); Rolf.Warta@med.uni-heidelberg.de (R.W.); Peter.Plinkert@med.uni-heidelberg.de (P.K.P.)

2 Heidelberg Institute of Global Health, University of Heidelberg, 69120 Heidelberg, Germany; Elisabethrudolph@web.de (E.R.); Heribert.Ramroth@uni-heidelberg.de (H.R.)

* Correspondence: Gerhard.Dyckhoff@med.uni-heidelberg.de; Tel.: +49-(0)-6221/56-6705

Citation: Dyckhoff, G.; Warta, R.; Herold-Mende, C.; Rudolph, E.; Plinkert, P.K.; Ramroth, H. An Observational Cohort Study on 194 Supraglottic Cancer Patients: Implications for Laser Surgery and Adjuvant Treatment. Cancers 2021, 13, 568. https://doi.org/10.3390/ cancers13030568

Academic Editor: Boban M. Erovic Received: 13 December 2020

Accepted: 28 January 2021

Published: 2 February 2021

Publisher's Note: MDPI stays neutral with regard to jurisdictional claims in published maps and institutional affiliations.

Copyright: (c) 2021 by the authors. Licensee MDPI, Basel, Switzerland. This article is an open access article distributed under the terms and conditions of the Creative Commons Attribution (CC BY) license (https:// creativecommons.org/licenses/by/ $4.0 /)$.
Simple Summary: Supraglottic laryngeal cancer patients suffer from a much poorer prognosis than patients with carcinoma arising from the glottis. Outstanding outcomes after laser surgical resection of early-stage supraglottic cancers have been reported by laser surgery centers of excellence. The aim of our retrospective observational cohort study was to assess whether similar results are achieved in the multi-institutional setting of less specialized facilities. We confirmed comparable oncological outcomes in five normal academic teaching hospitals, with a 5-year overall survival rate of $62.0 \%$ compared to $59.4-76.0 \%$ in the centers of excellence. In the case of microscopic residual tumors after surgical resection (R1) and/or lymph node metastases, adjuvant irradiation is recommended. Our data show that irradiation alone is not sufficiently effective in supraglottic cancers. Thus, for this tumor entity, adjuvant chemoradiation could be recommended.

Abstract: Supraglottic laryngeal cancer is characterized by poor prognosis. In contrast, excellent outcomes have been published in early-stage supraglottic cancers after laser surgery in singleinstitutional series in centers of excellence. Are these results reproducible in the normal clinical practice of less specialized facilities? As part of an observational cohort study, the outcomes of 194 supraglottic cancer patients were assessed after treatment by larynx-preserving surgery (transoral laser microsurgery [TLM] or open partial laryngectomy [OPL]) or total laryngectomy (TL), with each having risk-adopted adjuvant treatment, or primary (chemo-)radiotherapy (pCRT or pRT). In earlystage supraglottic cancers, TLM achieved a 5-year overall survival (5-year OS) of $62.0 \%$. No significant survival difference could be discerned between patients with and without adjuvant treatment (HR 1.47; 95\% CI: 0.80 2.69). The comparison between pCRT and pRT patients suggests that CRT is more effective in supraglottic cancer. The 5-year OS rate achieved in our multiinstitutional setting is comparable to that reached in laser surgery centers of excellence (59.4-76.0\%). According to our data and supported by the literature, adjuvant RT (aRT) is not sufficiently effective in supraglottic cancers. In case adjuvant therapy is indicated, adjuvant chemoradiation (aCRT) could be recommended.

Keywords: laryngeal cancer; supraglottic; larynx preservation; transoral laser microsurgery; radiotherapy; chemoradiation; adjuvant therapy

\section{Introduction}

Laryngeal cancer is the most common head and neck carcinoma which, in turn, is the sixth most common cancer worldwide, with 177,400 new cases per year. The agestandardized (worldwide) incidence rates vary strongly between the sexes, with 3.6 and 0.5 new cases per 100,000 per year, respectively, and the age-standardized mortality rates 
are 1.9 and 0.3 per 100,000 in men and women, respectively [1]. Laryngeal cancers can be anatomically subdivided into glottic, supraglottic and subglottic cancers. A total of $60-70 \%$ originate from the glottis, and approximately $35 \%$ from the supraglottic site [2], supraglottic cancer occurs more frequently in women [3,4]. The main risk factors are smoking of tobacco and excessive intake of alcohol $[5,6]$, with an even higher effect of alcohol in the supraglottic site [5,7]. As consistently shown, the outcome of supraglottic cancer is significantly poorer than that of glottic cancer. The 5-year overall survival (5-year OS) rate for localized disease is approximately $45-50 \%$ in supraglottic cancer compared to $85-90 \%$ in glottic cancer, indicating a $30-40 \%$ poorer 5 -year OS in tumors of the supraglottis $[4,8]$. The poorer prognosis is thought to be a consequence of abundant lymphatic drainage of the supraglottic region, resulting in higher rates of regional and distant metastases $[9,10]$. Moreover, cancers of the glottis often cause hoarseness and thus are relatively often diagnosed at an early stage, whereas supraglottic and subglottic cancers more often present with nodal involvement at the time of diagnosis [11]. For the treatment of early-stage supraglottic cancers, the National Comprehensive Cancer Network (NCCN) guidelines recommend single-agent therapy with either larynx-preserving surgery alone (endoscopic resection or open partial laryngectomy) or definitive radiotherapy alone [12].

We report the oncological outcomes of the subgroup of supraglottic laryngeal cancers of a cohort of index laryngeal cancer patients of a defined study region in southwest Germany. Patients were treated using surgical or conservative treatment modalities according to the standards of the five independent centers of the region.

\section{Results}

In total, 810 index laryngeal cancer patients were recruited for this cohort. Fifty-three patients were excluded because they either presented with synchronous second primary disease at first diagnosis $(n=12)$, received no treatment with curative intent $(n=28)$, or had an unknown tumor stage $(n=13)$. In the remaining 757 patients, there were 465 glottic $(64 \%), 194$ supraglottic $(26 \%), 14$ subglottic $(2 \%)$, and 51 transglottic cancers $(7 \%)$ and 33 patients with unknown localization of the primary tumor (4\%). The percentage of women was higher in supraglottic (14\%) than in glottic cancers $(8 \%)$. The median age in supraglottic cancers was 60 years compared to 63 years in glottic cancers. The tumor stage at primary diagnosis was comparatively higher in supraglottic carcinoma (T1: 19\%, T2: 35\%, T3: 27\%, and T4: 19\%) than in glottic carcinoma (T1: 63\%, T2: 25\%, T3: $9 \%$, and T4: $4 \%)$. Among supraglottic cancer patients, $104(54 \%)$ were treated by larynx-preserving surgery $(n=96[49.5 \%]$ TLM, and $n=7[3.6 \%]$ OPL) $n=57(29.4 \%)$ by TL, and $n=34(17.5 \%)$ by primary conservative treatment $(n=20[10.3 \%] \mathrm{pCRT}$, and $n=14[7.2 \%] \mathrm{pRT})$. The median follow-up was 8.4 years (0.04-16.8 years) and included patients with recurrence and death. Table 1 gives a demographic and clinical overview of the supraglottic tumor patients within the different treatment arms.

Table 1. Demographic and clinical characteristics of 194 supraglottic cancer patients.

\begin{tabular}{|c|c|c|c|c|c|c|c|}
\hline Variable & Category & TLM & OPL & TL & pCRT & pRT & All \\
\hline Total & & 96 & 7 & 57 & 20 & 14 & 194 \\
\hline Age (cont.) ${ }^{a}$ & & $59.6(37-79)$ & $61.3(51-77)$ & $59.7(40-80)$ & $58.4(41-77)$ & $62.1(40-79)$ & $59.7(37-80)$ \\
\hline \multirow[t]{2}{*}{ Sex } & Males & $81(84.4)$ & $7(100)$ & $53(93.0)$ & $15(75.0)$ & $10(71.4)$ & $166(85.6)$ \\
\hline & Females & 15 (15.6) & $0(0.0)$ & $4(7.0)$ & $5(25.0)$ & $4(28.6)$ & 28 (14.4) \\
\hline \multirow[t]{2}{*}{$\mathrm{CCI}$} & 0 & $71(74.0)$ & $4(57.1)$ & $38(66.7)$ & $16(80.0)$ & $5(35.7)$ & $134(69.1)$ \\
\hline & 1 & $25(26.0)$ & $3(42.9)$ & 19 (33.3) & $4(20.0)$ & $9(64.3)$ & $60(30.9)$ \\
\hline \multirow[t]{4}{*}{ T category } & 1 & $31(32.3)$ & $1(14.3)$ & $1(1.8)$ & $2(10.0)$ & $2(14.3)$ & 37 (19.1) \\
\hline & 2 & 39 (40.6) & $4(57.1)$ & $14(24.6)$ & $5(25.0)$ & $6(42.9)$ & $68(35.1)$ \\
\hline & 3 & $16(16.7)$ & $2(28.6)$ & $24(42.1)$ & $7(35.0)$ & $3(21.4)$ & $52(26.8)$ \\
\hline & 4 & $10(10.4)$ & $0(0.0)$ & 18 (31.6) & $6(30.0)$ & $3(21.4)$ & 37 (19.1) \\
\hline
\end{tabular}


Table 1. Cont.

\begin{tabular}{|c|c|c|c|c|c|c|c|}
\hline Variable & Category & TLM & OPL & TL & pCRT & pRT & All \\
\hline \multirow[t]{5}{*}{ N stage } & 0 & $50(52.1)$ & $5(71.4)$ & $30(52.6)$ & $9(45.0)$ & $4(28.6)$ & $98(50.5)$ \\
\hline & 1 & 15 (15.6) & $0(0.0)$ & 7 (12.3) & $2(10.0)$ & $3(21.4)$ & 27 (13.9) \\
\hline & 2 & $26(27.1)$ & $2(28.6)$ & $20(35.1)$ & $6(30.0)$ & $6(42.9)$ & 60 (30.9) \\
\hline & 3 & $1(1.0)$ & $0(0.0)$ & $0(0.0)$ & $2(10.0)$ & $1(7.1)$ & $4(2.1)$ \\
\hline & $x$ & $4(4.2)$ & $0(0.0)$ & $0(0.0)$ & $1(5.0)$ & $0(0.0)$ & $5(2.6)$ \\
\hline \multirow[t]{4}{*}{ UICC stage } & $\mathrm{I}$ & $20(20.8)$ & $0(0.0)$ & $1(1.8)$ & $1(5.0)$ & $0(0.0)$ & $22(11.3)$ \\
\hline & II & $20(20.8)$ & $4(57.1)$ & 7 (12.3) & $3(15.0)$ & $4(28.6)$ & 38 (19.6) \\
\hline & III & $23(24.0)$ & $1(14.3)$ & $19(33.3)$ & $6(30.0)$ & $3(21.4)$ & $52(26.8)$ \\
\hline & IV & $33(34.4)$ & $2(28.6)$ & $30(52.6)$ & $10(50.0)$ & $7(50.0)$ & $82(42.3)$ \\
\hline \multirow[t]{2}{*}{ Neck diss } & No & $25(26.0)$ & $1(14.3)$ & $3(5.3)$ & $18(90.0)$ & $14(100)$ & $61(31.4)$ \\
\hline & Yes & $71(74.0)$ & $6(85.7)$ & $54(94.7)$ & $2(10.0)$ & $0(0.0)$ & $133(68.6)$ \\
\hline \multirow[t]{5}{*}{ Resection } & 0 & 39 (40.6) & $6(85.7)$ & 38 (66.7) & $0(0.0)$ & $0(0.0)$ & $83(42.8)$ \\
\hline & 1 & $18(18.8)$ & $0(0.0)$ & $7(12.3)$ & $0(0.0)$ & $0(0.0)$ & 25 (12.9) \\
\hline & 2 & $4(4.2)$ & $0(0.0)$ & $0(0.0)$ & $0(0.0)$ & $0(0.0)$ & $4(2.1)$ \\
\hline & $X$ & $28(29.2)$ & $1(14.3)$ & $9(15.8)$ & $2(10.0)$ & $3(21.4)$ & $43(22.2)$ \\
\hline & missing & $7(7.3)$ & $0(0.0)$ & $3(5.3)$ & $18(90.0)$ & $11(78.6)$ & $39(20.1)$ \\
\hline \multirow[t]{5}{*}{ Adj. ther. } & None & $49(51,0)$ & $4(57.1)$ & $26(46)$ & n.a. & n.a. & $79(40.1)$ \\
\hline & aRT & $43(44.8)$ & $3(42.8)$ & $23(41.1)$ & n.a. & n.a. & $69(35.6)$ \\
\hline & aCRT & $4(4.1)$ & $0(0.0)$ & 7 (12.5) & n.a. & n.a. & $11(5.7)$ \\
\hline & $\mathrm{aCT}$ & $2(2.0)$ & $0(0.0)$ & $0(0.0$ & n.a. & n.a. & $2(1.0)$ \\
\hline & unknown & $14(14.6)$ & $0(0.0)$ & $5(8.8)$ & $2(10.0)$ & 2 (14.3) & $23(11.9)$ \\
\hline
\end{tabular}

a Mean (Standard Deviation); Age (cont.) = Age (continuous); Neck diss = Neck dissection; Resection = Resection status; Adj. ther. = Adjuvant therapy; n.a. = not applicable.

Early-stage (T1 and T2) supraglottic cancers after laser surgery showed a significantly worse OS than early glottic cancers (HR adjusted for age and comorbidities: 1.78, 95\% CI:1.27-2.49; $p=0.0009$ ) (Figure 1). The 5-year and 10-year disease specific survival (DSS) and overall survival (OS) Kaplan-Meier estimates and the corresponding 95\% CIs of earlystage supraglottic and glottic cancers are shown in Table 2.

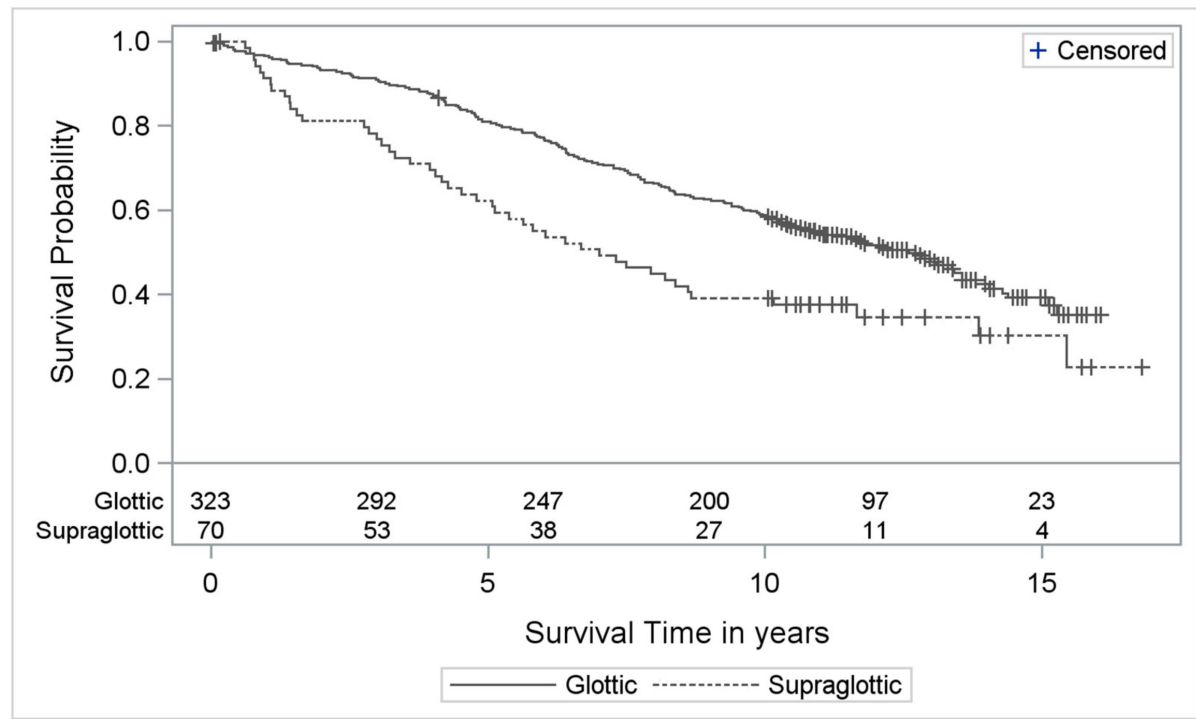

Figure 1. Kaplan Meier curves with numbers at risk of supraglottic compared to glottic cancers $(\mathrm{T} 1+\mathrm{T} 2)$ treated by TLM $(\mathrm{OS})$ (solid line = glottic; dashed line = supraglottic $)$. 
Table 2. 5-year and 10-year DSS and OS of early-stage supraglottic and glottic cancers after TLM.

\begin{tabular}{|c|c|c|c|c|}
\hline $\begin{array}{l}\text { Localization, T } \\
(n, \mathrm{DSS} / \mathrm{OS}) \text { * }\end{array}$ & $\begin{array}{c}\text { 5-y DSS [\%] } \\
(95 \% \mathrm{CI})\end{array}$ & $\begin{array}{c}\text { 10-y DSS [\%] } \\
(95 \% \mathrm{CI})\end{array}$ & $\begin{array}{c}\text { 5-y OS [\%] } \\
(95 \% \text { CI) }\end{array}$ & $\begin{array}{c}\text { 10-y OS [\%] } \\
(95 \% \mathrm{CI})\end{array}$ \\
\hline $\begin{array}{l}\text { Supraglottic } \\
\text { T1 }(29 / 31)\end{array}$ & $92(71-98)$ & 87 (65-96) & 65 (45-79) & $32(17-49)$ \\
\hline T2 (39/39) & $75(57-86)$ & $71(53-83)$ & $61(43-74)$ & $45(29-60)$ \\
\hline $\mathrm{T} 1+\mathrm{T} 2(68 / 70)$ & $82(69-89)$ & $78(64-86)$ & $62(50-73)$ & $39(28-50)$ \\
\hline $\begin{array}{l}\text { Glottic } \\
\qquad \text { T1 }(238 / 243)\end{array}$ & $96(93-98)$ & $94(90-97)$ & $82(76-86)$ & $60(54-66)$ \\
\hline $\mathrm{T} 2(80 / 80)$ & 89 (80-95) & $82(70-89)$ & $79(68-86)$ & $54(42-64)$ \\
\hline $\mathrm{T} 1+\mathrm{T} 2(318 / 323)$ & 95 (91-97) & $91(87-94)$ & $81(76-85)$ & $59(53-64)$ \\
\hline
\end{tabular}

${ }^{*}$ Differences in numbers of patients between OS and DSS due to unknown causes of death.

Histopathologically clear resection margins (R0) were documented in $40.6 \%$, tumor ramifications were microscopically described in $18.8 \%$ (R1), tumor was retained macroscopically (R2) in four patients (4.2\%), definite R classification could not be established in $29.2 \%(\mathrm{Rx})$, and the resection status was missing in the charts of six patients (Table 3 ). A multivariable Cox regression model did not show any significant survival difference between uncertain margins (Rx) and histopathologically free margins (R0) (HR 1.41; 95\% CI: $0.72-2.78$ ) or between positive resection margins (R1, R2) and R0 (HR 0.71; 95\% CI: 0.35-1.48).

Table 3. Resection status after laser surgery over T category.

\begin{tabular}{cccccc}
\hline T Category & R0 & Rx & R1 & R2 & R Missing \\
\hline T1 $(n=31)$ & $14(45.2 \%)$ & $10(32.3 \%)$ & $5(16.1 \%)$ & $0(0.0 \%)$ & $2(6.5 \%)$ \\
T2 $(n=39)$ & $20(51.3 \%)$ & $8(20.5 \%)$ & $9(23.1 \%)$ & $0(0.0 \%)$ & $2(5.1 \%)$ \\
T3 $(n=16)$ & $4(20.5 \%)$ & $8(50.0 \%)$ & $2(12.5 \%)$ & $1(6.3 \%)$ & $1(6.3 \%)$ \\
T4 $(n=10)$ & $2(20.0 \%)$ & $2(20.0 \%)$ & $3(30.0 \%)$ & $2(20.0 \%)$ & $1(10.0 \%)$ \\
\hline
\end{tabular}

In the TLM group, aRT was administered in $43(44.8 \%)$ patients, aCRT in $4(4.2 \%)$, aCT in $2(2.1 \%)$ and no adjuvant treatment in $47(48.9 \%)$ patients. The distribution of the $\mathrm{T}$ category is given in Table 4 .

Table 4. Adjuvant treatment after TLM over T category. No adj. = No adjuvant therapy.

\begin{tabular}{ccccc}
\hline T Category & No adj. $(n=47)$ & aRT $(\boldsymbol{n}=\mathbf{4 3})$ & aCRT $(\boldsymbol{n}=\mathbf{4})$ & aCT $(\boldsymbol{n}=\mathbf{2})$ \\
\hline T1 $(n=31)$ & $23(74.2 \%)$ & $7(22.6 \%)$ & $0(0 \%)$ & $1(3.2)$ \\
T2 $(n=39)$ & $14(35.9 \%)$ & $24(61.5 \%)$ & $1(2.6 \%)$ & $0(.0)$ \\
T3 $(n=16)$ & $8(50.0 \%)$ & $6(37.5 \%)$ & $1(6.3 \%)$ & $1(6.3)$ \\
T4 $(n=10)$ & $2(20.0 \%)$ & $6(60.0 \%)$ & $2(20.0 \%)$ & $0(0.0)$ \\
\hline
\end{tabular}

The procedural characteristics of neck dissection, resection status, and adjuvant treatment based on UICC stage are given in Table 5.

Table 5. Procedural characteristics by stage.

\begin{tabular}{ccccccc}
\hline Procedure & Category & Stage I & Stage II & Stage III & Stage IV & All \\
\hline ND & No & $14(63.6)$ & $15(39.5)$ & $15(28.8)$ & $17(20.7)$ & $61(31.4)$ \\
& Yes & $8(36.4)$ & $23(60.5)$ & $37(71.2)$ & $65(79.3)$ & $133(68.6)$ \\
Resection & R0 & $10(45.5)$ & $21(55.3)$ & $24(46.2)$ & $28(34.1)$ & $83(42.8)$ \\
& Rx/R1/R2 & $11(50.0)$ & $9(23.7)$ & $15(28.8)$ & $37(45.1)$ & $72(37.1)$ \\
Adj. treatment & Missing & $1(4.5)$ & $8(21.1)$ & $13(25.0)$ & $17(20.7)$ & $39(20.1)$ \\
& No & $21(95.5)$ & $29(76.3)$ & $28(53.8)$ & $34(41.5)$ & $112(57.7)$ \\
& Yes & $1(4.5)$ & $9(23.7)$ & $24(46.2)$ & $48(58.5)$ & $82(42.3)$ \\
\hline
\end{tabular}


In a multivariable Cox regression model, no significant survival differences could be discerned between patients with and without adjuvant treatment (HR 1.47; 95\% CI: 0.80-2.96). As an example of the evaluation matrix of radiation efficacy (definitions and set-up see Material and Methods) the results for T2 patients with clear indication for adjuvant therapy is shown in Table 6.

Table 6. Success and failure, respectively, after aRT in T2 supraglottic cancer patients with a clear indication for adjuvant treatment. Each patient defined by his resection $(\mathrm{R})$ and lymph node $(\mathrm{N})$ status in bold letters, followed by OS [y=years, $\mathrm{m}=$ months], and cause of death; LT-S = long-term survivor, i.e., living at the end of follow-up time; tu = death due to locoregional recurrent tumor; NSCLC = Non-small cell lung cancer; Rect-Ca = Carcinoma of the rectum; Colon-Ca = Colon cancer; Pneumo = Pneumonia .

\begin{tabular}{|c|c|c|c|}
\hline Indication & Success & Intermediate/Uncertain & Failure \\
\hline 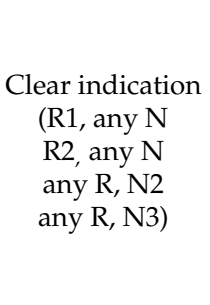 & 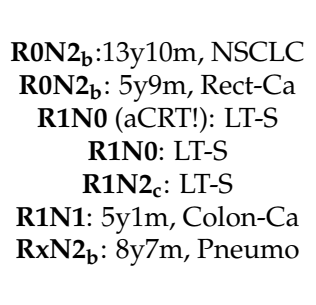 & $(\mathbf{R} 0 \mathrm{~N} 2 \mathbf{b}): 4 y 3 \mathrm{~m} \mathrm{M} 1$ & $\begin{array}{r}\text { R0N2 }: 3 y 2 m, t u \\
\text { R0N2: } 2 y 11 \mathrm{~m}, \mathrm{tu} \\
\text { R0N2 }{ }_{\mathrm{c}}: 1 \mathrm{y} 5 \mathrm{~m}, \mathrm{tu} \\
\text { R1N0: } 0 \mathrm{y} 9 \mathrm{~m} \mathrm{tu} \\
\text { R1N2 } \\
\text { R1N2: } 2 \mathrm{y} 10 \mathrm{~m}, \mathrm{tu} \\
\text { R1N1: } 1 \mathrm{y} 3 \mathrm{~m}, \mathrm{tu} \\
\text { RxN2: } 1 \mathrm{y} 3 \mathrm{~m}, \mathrm{tu} \\
\text { RxN3: } 1 \mathrm{y} 5 \mathrm{~m}, \mathrm{tu}\end{array}$ \\
\hline
\end{tabular}

For the sake of maximum power without restriction to TLM, a multivariable Cox regression model of the whole cohort of 194 supraglottic cancer patients was performed comparing the outcome of all surgically treated patients (TLM, OPL, and TL) with that of conservative patients. There was a significantly worse outcome after pRT alone (HR 2.39; 95\% CI: 1.33-4.29) but there was no significant difference between pCRT and surgery (HR 1.53; 95\% CI: 0.93 2.52) (Figure 2).

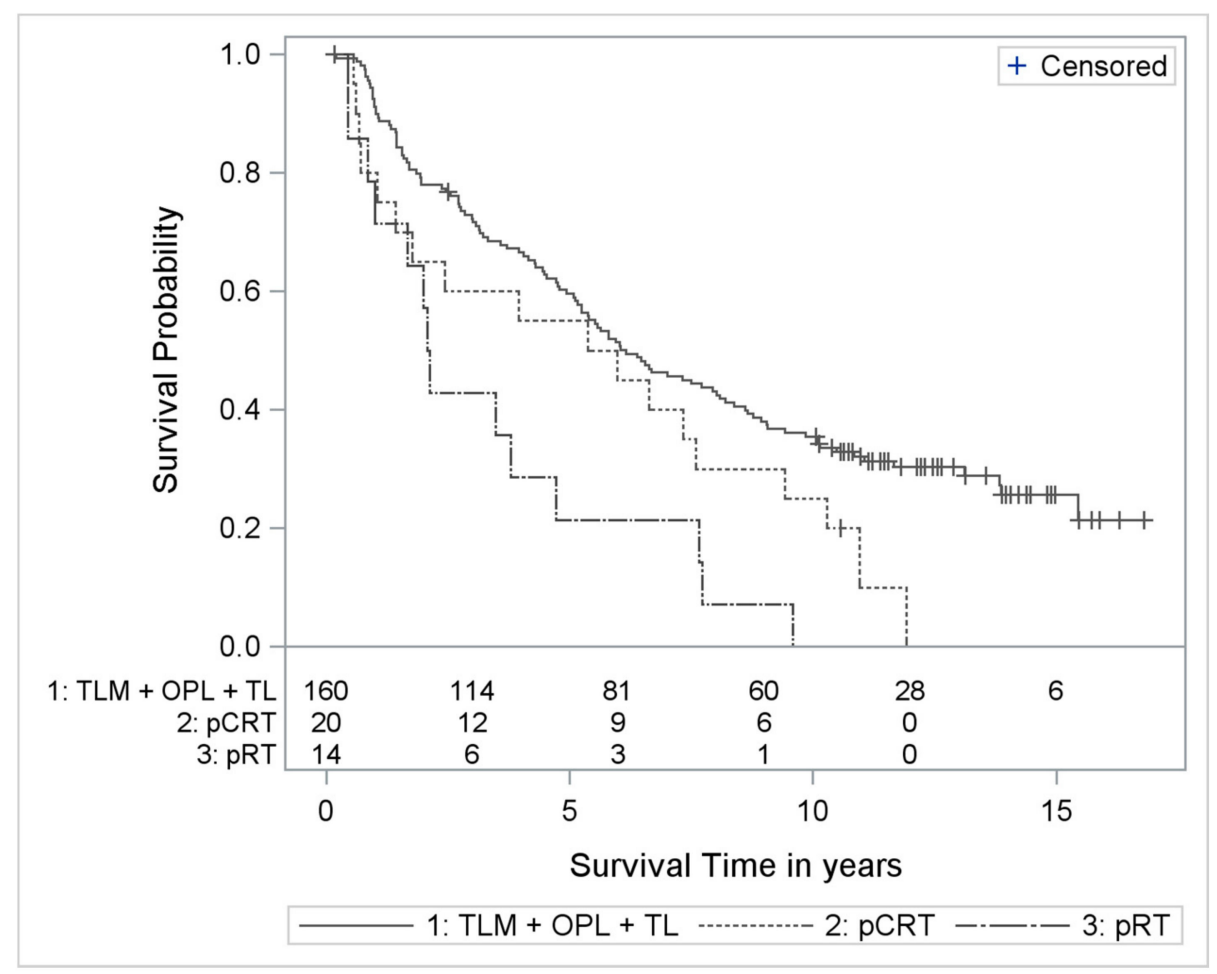

Figure 2. Kaplan Meier curves with numbers at risk of supraglottic cancers treated with pCRT or pRT compared to TLM + OPL + TL (OS). 


\section{Discussion}

As consistently shown, supraglottic cancers have a poorer prognosis than glottic cancers $[4,8,13]$. As an example, Silvestri reported a 3-year adjusted survival rate of $46 \%$ for supraglottic carcinoma patients compared to $83 \%$ for glottic carcinoma patients [13]. One reason is that glottic tumors are diagnosed at an earlier stage than tumors of the supraglottic site, as hoarseness, the cardinal symptom of glottic carcinoma, occurs with few lesions of the vocal folds. In our cohort, $62.4 \%$ of glottic tumors were diagnosed as early as stage I, compared to no more than $18.6 \%$ in supraglottic cancers. This is consistent with Raitiola, who found $51.2 \%$ stage I glottic tumors compared to $14.2 \% \mathrm{~T} 1$ tumors arising from the supraglottis [11]. A second important reason is that supraglottic cancers more often present with nodal involvement at the time of diagnosis $[8,11,13]$. This is ascribed to the richer lymphatic supply of the supraglottic area $[9,10]$. Thus, even in T1 and T2 supraglottic carcinomas, treatment of the neck has to be considered. In our cohort, $55.3 \%$ of T1 patients and $70.0 \%$ of $\mathrm{T} 2$ patients received neck dissection. A multivariable Cox regression model also revealed a poorer prognosis for supraglottic cancers in our cohort. Focusing on early-stage, we found a significantly worse OS after TLM in supraglottic cancers than in glottic carcinomas (HR 1.78, 95\% CI:1.27-2.49; $p=0.0009$ ) (Figure 1). Iro et al. published a comparable difference in outcome (5-year OS of $59.4 \%$ in supraglottic tumor patients compared to $77.7 \%$ in glottic carcinoma patients) [14].

After important pioneering work was performed by Vaughan and Davis using a carbon dioxide $\left(\mathrm{CO}_{2}\right)$ laser for resection of benign and malignant lesions of the supraglottis first $[15,16]$, Steiner systematically introduced TLM for the curative treatment of supraglottic cancers in Germany [17]. As an excellent surgeon and teacher, he established the laser surgery schools of Goettingen and Erlangen-Nuremberg, which performed several single institution series with outstanding results $[14,17-20]$. A similar outcome was achieved by Davis in Salt Lake City (UT, USA) [21]. The question is whether these results are representative of TLM in the multiinstitutional setting of less specialized centers. As an answer, we report the outcomes of laser surgery in supraglottic tumors as part of a retrospective observational cohort study of all index laryngeal cancer patients of a defined study region who were treated using surgical or conservative treatment modalities according to the standards of five independent academic teaching hospitals. The 5-year and 10-year OS rates were $65 \%$ and $32 \%$ in $\mathrm{T} 1$ and $61 \%$ and $45 \%$ in $\mathrm{T} 2$ supraglottic cancer patients, respectively. In most published studies in the literature, OS is presented for early stage (T1 and T2) patients together and mostly as 5-year OS. The 5-year OS of our pooled T1 and T2 patients $(n=70)$ amounted to $62.0 \%$. In Table 7 , the 5 -year OS of our observational multicenter study fits the results achieved by the centers of excellence in which the OS rate ranges from $59.4 \%$ to $76 \%$. Notably, the OS reported for combined stage I + II disease is probably better than the OS reported for $\mathrm{pT} 1+\mathrm{pT} 2$ patients. This is because in the stage I + II group, all pT1 and pT2 tumor patients, who suffered from cervical lymph node metastases and thus were classified stage III or IV, were excluded.

Table 7. 5-year OS of early-stage supraglottic cancer patients after TLM in different studies.

\begin{tabular}{ccc}
\hline Study & Number of Patients & 5-y OS [\%] \\
\hline Ambrosch, 1998 [17] & 48 & $76.0\left(\mathrm{pT}_{1}+\mathrm{T}_{2}\right)$ \\
Iro, 1998 [18] & 69 & $75.4($ stage I + II) \\
Steiner, Ambrosch, 1996 [22] & 43 & $72.8($ stage I + II) \\
Ambrosch, 2018 [20] & 67 & $69.0($ stage I + II) \\
Dyckhoff, present study & 70 & $62.0\left(\mathrm{pT}_{1}+\mathrm{pT}_{2}\right)$ \\
Iro, 2011 [14] & 137 & $59.4\left(\mathrm{pT}_{1}+\mathrm{pT}_{2}\right)$ \\
\hline
\end{tabular}

A direct comparison of some further important work is not possible because only 3-year, but not 5-year OS was given (e.g., Rudert et al.: 17 patients T1 + T2: 3-year OS: $88 \%$ [23]), or higher stage tumors were included (e.g., Davis: 46 cT2 patients, $39 \%$ histopathologically restaged from cT2 to $\mathrm{pT} 3$ because of preepiglottic space infiltration, 
5-year OS: 63\% [21]). This work, however, fits in the range of outcomes published by the other centers of excellence. From the epidemiological point of view, a direct comparison of these studies was prohibited, as the Kaplan-Meier estimates are univariate and do not consider differences in the composition of the respective cohorts as to age, comorbidities, or other confounders. Compared with the other publications, our study is the only one on supraglottic cancers providing long-term results after laser surgery with 10-year OS data.

While the oncological outcomes are comparable, great discrepancies can be seen between the results of our five regional facilities taking part in our observational cohort study and the results of the single-institution series published by centers of excellence when considering the resection status. In 2018, Ambrosch reported outstanding 90.1\% successful R0 resections with one single positive microscopic tumor ramification. In only $8.8 \%(n=8)$, definite R classification could not be established [20]. In 2011, Iro et al. reached the same excellent result of $91.2 \% \mathrm{R} 0$ resections and only $8.8 \% \mathrm{R}+$. The number of $\mathrm{R}+$ patients was too low to enable statistical interpretation in the form of log-rank $p$-values [14]. These outstanding results were reached in centers of excellence by highly experienced and very well-trained laser surgeons. Iro emphasizes the importance of certain R0 resection to achieve satisfactory oncological results [18]. Optimal exposure and visualization of the tumor is necessary for safe tumor resection, as Steiner and Ambrosch point out in their description of the operative technique [17]. Of exceeding importance for proper histopathological assessment of the resection margins is the careful orientation and accurate mapping of the resected specimens, especially if the resection has to be performed in several blocks (usually necessary in advanced tumor stage) [24]. Histopathological examination must be performed by serial sections. For the achievement of accurate $\mathrm{pT}$ staging, it must finally be possible for the surgeon "to recreate a complete 3-dimensional mosaic of the entire lesion" [24]. If no clear R0 resection is reached, reresection is necessary. If this is not possible the switch to transcervical open surgery or even TL is indicated $[17,18,21]$.

In contrast to these outstanding results of up to over $90 \% \mathrm{R} 0$ resection and Rx of less than $10 \%$ in the centers of excellence, the evaluation of our multicenter study revealed histopathologically proven R0 resections in only half of the cases (T1: $45 \%$, and T2 51\%), while there was a large number of $\mathrm{Rx}(\mathrm{T} 1: 32 \%$, and $\mathrm{T} 2: 21 \%$ ) and $\mathrm{R} 1$ resections (T1: $16 \%$, and T2: $23 \%$ ). However, multivariable Cox regression analysis revealed no significant difference in outcome between uncertain margins (Rx) and histopathologically free margins (R0) (HR 1.41; 95\% CI: 0.72-2.78), or between positive margins (R1, R2) and R0 (HR 0.71; 95\% CI: 0.35-1.48). This is in accordance with a recent database study of Jumaily et al., who analyzed 747 T1-2 glottic carcinomas treated by TLM. 5-year OS for patients with positive margins was lower $(80.0 \%)$ than that with negative margins $(82.9 \%)$, but without statistical significance in multivariate analysis $(p=0.96)$. The rate of positive resection margins in his study was $19.9 \%$ [25]. Regarding the more favorable survival data, it should be noted that the study was conducted on glottic carcinomas and $89.1 \%$ of patients presented with T1 tumors. In a second recent database study on 1959 TIS-T3 laryngeal cancer patients (of which $21.3 \%$ were supraglottic), Hanna reported $31.3 \%$ positive margins in supraglottic cancers compared to $19.7 \%$ in glottic tumors, without showing statistical significance in multivariable analysis [26]. However, no survival data were presented. In a series of 203 laryngeal cancer patients with 39\% supraglottic tumors, Pedregal-Mallo reported 17\% positive margins in supraglottic tumors compared to $2 \%$ in glottic cancers [27]. In his study, involvement of surgical margins was associated with lower disease-specific survival in multivariable analysis $(p=0.004)$ [27]. Interestingly, in the large database studies of Hanna and Jumaily no Rx or uncertain margins were reported [25,26]. Ambrosch and Steiner, however, point out that this is a realistic situation to face even when following the highest standards. If tumor infiltration reaches cartilage or bony structures, clear R0 resection may be impossible to prove; in these cases, an Rx status cannot be omitted [24]. Thus, in most European studies beside R1, R2, and R0 a certain amount of Rx is also reported. The question is whether " $\mathrm{Rx} /$ uncertain resection margin" was actively captured in the National Cancer database. If an information specialist entering data can only choose 
between R0 and R1 but a resection margin is uncertain, documentation bias can occur. Therefore, the information given by Hanna is extremely interesting: only $55 \%$ of patients with positive margins received adjuvant radiation [26]. Follow-up of the other $45 \%$ could prove the correct assessment of positive margins (R1). In true residual tumors, recurrence and tumor related death are likely to occur. While Rx seems to be missing in these American studies, the Italian group of Fiz et al. used a sophisticated R classification system with 6 categories to differentiate between negative, close superficial (i.e., $<1 \mathrm{~mm}$ ), close deep, positive single superficial, positive multiple superficial, and positive deep [28]. Using this system, only 232 out of 507 glottic cancer patients were negative $(45.8 \%)$, while the rest were close margin or positive. In this differentiated system, an undifferentiated Rx status appears but superfluous. The authors show that there is a significant correlation between the different forms of positive margins and recurrence-free survival. Interestingly, this study did not follow Steiner's dogma that the paramount aim of laser surgery is achieving histopathologically free margins. Patients with single positive superficial margins-the same as with close margins-were strictly followed-up but not reresected. Only in positive multiple superficial or deep margins was further treatment (TLM reresection, OPL, or aRT) proposed to the patient. Using the recurrence-free survival (RFS) of $89.4 \%$ of the pooled cases of negative and close margin cases as a reference, single superficial had an RFS of $83.3 \%(p=\mathrm{NS})$, multiple superficial $72.7 \%(p<0.001)$ and deep positive margins $75.8 \%$ $(p<0.01)$ [28].

In 1998, with the beginning of systematic TLM in supraglottic cancer, Iro published the first series with an R0 resection rate in T1 and T2 supraglottic tumors of $88.4 \%$ (61/69 patients) in a study of 141 patients with an overall R0 resection rate of $78 \%$ [18]. With the experience of the corresponding $\mathrm{R}+$ patients, Iro reported a clear correlation between resection status and survival. With a value of $p<0.001$, the difference in survival between patients with negative margins (R0) and those with positive margins despite receiving aRT, reached a high significance level (OS of 76.4\% compared to 17.8\%) [18]. The question remains why there was no statistical correlation between poor resection status and survival - neither OS nor DSS - in our multiinstitutional study. Furthermore, the patients in our study with an $\mathrm{R} 0$ resection rate of $48 \%$ had a survival outcome of $62 \%$, which is comparable to the $59.4 \%$ in the study of Iro, in which an R0 resection was achieved in more than $90 \%$ of cases. Meanwile, in Iro's study, there was a statistically significant correlation between resection status and outcome. The solution might be found in the Rx resections. According to Ambrosch and Steiner, Rx status is properly assessed in rare cases in which deep tumor infiltration reaches cartilage or bony structures. However, in all other cases, after properly applying Steiner's technique of careful orientation and accurate mapping of the resected specimen "to recreate a complete 3-dimensional mosaic of the entire lesion," a distinct R0 or R1 resection should be possible [24]. If fresh frozen sections reveal residual tumor, the resection has to continue until clear margins are reached and the pathologist reports no more ramification in the last properly oriented specimen. It could be assumed that this meticulous work of careful orientation, accurate mapping and recreating the mosaic is not done in the same scrupulous way in less specialized facilities as in a center of excellence. Therefore, resection may be performed in an excellent manner, reaching clear margins with consequent favorable survival outcomes-but the histopathological results do not reflect the surgical results, as the definite $\mathrm{R}$ classification cannot be established, and is thus called " $\mathrm{Rx}$ ".

On multivariable analysis, Hanna reported factors associated with positive margins. For T-stage T3 he found the highest negative impact with an HR of 5.53 (3.55-8.63) [26]. In parallel, in the T3 tumors of our cohort we reached R0 resection in only half of the number of cases compared to T1 and T2. In contrast, Hanna also found a smaller but still significant difference for T2 compared to T1 (HR 2.74 [2.05 3.65]), while the rate of R0 resection in our cohort was almost the same (T1: 45\%, T2: 51\%). A possible explanation might be found in the other negative impact factors described by Hanna: nonacademic vs. academic status, and lower caseload facilities had a higher likelihood of positive margins. 
Overall, $60.6 \%$ of patients reported in the National Database study were treated at facilities performing $<2$ cases per year [26]. In contrast, all the centers in charge of oncological therapy in our study region were academic teaching hospitals performing laser surgery on a regular basis. Thus, our results can be seen in accordance with Hanna's findings. Similarly, Ambrosch and Iro recommend TLM in supraglottic tumors as a good option for surgical larynx preservation for early supraglottic carcinomas (T1 and T2) but restrict this recommendation in locally advanced cancers-conforming to the current NCCN guideline [12]—-to "only selected cases" [14,20]. They state this as representatives of laser surgery centers of excellence. Our observational multicenter study shows that ordinary academic teaching hospitals can achieve comparable results. Thus, we agree with Hanna that for T3, the proper patient selection for TLM is important, but for T1 and T2, it is more important to select the appropriate facility. The consequence of Hanna's study should not be a change in the therapy concept but rather an improved training of laser surgeons and concentration of cases of supraglottic cancer in centers with sufficient experience and caseload, as proposed by Harréus [29]. Based on the experience of our study we would like more emphasis to be placed on the training and experience in laser surgery as well as on the accuracy in the processing of resected specimens because proper assessment of resection margins is required for the correct indication of reresection or adjuvant treatment.

In early-stage laryngeal cancer, the treatment of choice is single agent therapy, either surgical or nonsurgical. The paramount aim of the surgical approach is the complete removal of the tumor so that adjuvant therapy in node-negative disease is not necessary. Just as salvage surgery is an integral part of the conservative concept but primarily by no means intended, adjuvant treatment is a salvage option when the primary treatment goal is not reached, but further resection is not possible or not accepted by the patient. Thus, an adjuvant treatment rate of 51\% in a TLM group must raise concern. The procedural characteristics based on UICC stage show, however, that only one stage I patient $(4,5 \%)$ and nine stage II patients $(23.7 \%)$ received adjuvant treatment because of a lack of free margins (Table 5). Most of the patients received adjuvant treatment because locoregional advanced stages.

The distribution of primary treatment modalities clearly reveals that, in the respective region of Germany 20 years ago, there was a strong preference for primary surgery over $\mathrm{p}(\mathrm{C}) \mathrm{RT}$. This can be a source of bias, as patients with comorbidities, lower compliance or lack of social support might have been chosen for nonsurgical treatment. While the documented Charlson Comorbidity Index does not show this tendency, this possible bias could not be excluded in this retrospective study. Thus, one should exercise caution when comparing surgical and non-surgical treatment approaches. Moreover, the low number of patients treated conservatively compared to the large number treated by surgery makes a proper statistical comparison difficult. Refraining from inferential statistics concerning the outcome of TLM and pRT, valuable-even though preliminary-conclusions can be drawn concerning the effectiveness of radiotherapy alone compared to radiation with adjuvant chemotherapy. A Cox regression model comparing each-pCRT and pRT-to surgery showed an adjusted hazard ratio of 1.53 (95\% CI: 0.93-2.52) which is not significant for pCRT compared to a statistically significant HR of 2.39 (95\% CI: 1.33-4.29) for pRT. Since a direct comparison of pCRT and pRT is not reasonable because of the low number of cases, this allows, however, a cautious conclusion to be drawn concerning the superiority of CRT over RT in the entity of supraglottic cancer. Comparison of the Kaplan-Meier curves of the patients treated by pCRT and by pRT, descriptively illustrates the superiority of CRT over RT alone (Figure 2).

A multivariable Cox regression model revealed no significant survival difference between patients with and without adjuvant treatment (HR 1.46; 95\% CI: 0.80-2.69). In an attempt to understand what "missing effect of adjuvant treatment" means on a differentiated and personalized basis, we designed an evaluation matrix of adjuvant treatment. At first, we defined criteria for the assessment of success and failure of adjuvant treatment (see Materials and Methods, Table 8). Patients were then classified according to the 
safety degree of indication of adjuvant treatment (no indication, uncertain, probable, and clear indication according to R status and N stage following the NCCN guidelines [12] and categorized of one particular $\mathrm{T}$ stage in a bidimensional matrix according to safety of indication and success of adjuvant treatment in terms of oncological outcomes given by survival [years, months] and cause of death. As an example, we present the results of the T2 patients with clear indication of adjuvant treatment (R1, R2, and/or N > 1) in Table 6. A direct comparison of the aRT patients with oncological success and oncological failure shows at first sight, that for the very same oncological indication the failure-patients outweigh the success-patients ( $n=9$ vs. $n=7$ ). Considering the individual time to death by tumor ("tu") gives an impact of the necessity to find a more effective adjuvant treatment to possibly prevent this outcome. The result of our study is consistent with Iro, who reported that adjuvant irradiation did not show a significant effect on the prognosis of patients with $\mathrm{R}+$ resections $(p<0.001)$. The recurrence-free survival rate of patients in whom TLM did not induce an R0 condition was $17.8 \%$, compared to $76.4 \%$ in R0 patients despite aRT [18]. Thus, as aRT could not compensate for positive resection margins, Iro's consequence was to aim at R0 resection at all costs: either by (repeated) TLM reresection or by switch to OPL or-if necessary-by TL. If the patient did not agree to further surgery, there seemed to be no alternative. The data of our study, however, propose a different option: if adjuvant radiotherapy alone is ineffective in supraglottic cancer, efficacy can be augmented by the addition of chemotherapy.

Table 8. Differential matrix for the definition of success of adjuvant treatment.

Failure

Early failure: tumor-related death $\leq 2$ years

Intermediate failure: tumor related death 2 years $<\ldots \leq 5$ years

Late failure/success not sustained: tumor related death $>5$ years

\begin{tabular}{|c|c|c|}
\hline \multirow{2}{*}{ Intermediate/uncertain } & \multirow{2}{*}{ Uncertain success } & Death due to intercurrent disease, e.g., myocardial infarction, stroke $\leq 5$ years \\
\hline & & $\begin{array}{c}\text { Tumor-related death which cannot be prevented by locoregional treatment, } \\
\text { i.e., distant metastases } \leq 5 \text { years }\end{array}$ \\
\hline Success & $\begin{array}{l}\text { Sustained or } \\
\text { Clear suc }\end{array}$ & $\begin{array}{l}\text { gic success: no recurrence, death due to intercurrent disease }>5 \text { years } \\
\text { : no recurrence and long-term survivor (beyond time of follow-up) }\end{array}$ \\
\hline
\end{tabular}

For the definitive setting, chemoradiation is an established treatment concept for advanced stage disease. For the adjuvant setting, it has become standard since the large prospective studies of Cooper and Bernier [30,31]. With level I evidence, they have shown improved survival in high-risk patients with positive resection margins $(\mathrm{R}+)$ and/or extracapsular spread (ECS+) in lymph node metastases for head and neck cancers (HNSCC) in general. In Cooper's and Bernier's cohorts, most tumors were oropharyngeal carcinoma (in the combined therapy group $48 \%$ and $32 \%$, respectively), for which a high response rate for radiotherapy and especially chemoradiotherapy is well-documented. According to our data and supported by the literature, the entity of supraglottic cancer is not sufficiently responsive to adjuvant radiotherapy. In Cooper's study, there were $15 \%$ and $14 \%$ supraglottic cancers in each treatment arm [30], and 23\% and 22\% laryngeal cancers in Bernier's cohort, respectively, without explicitly mentioning the proportion of supraglottic cancers [31]. However, neither study showed specific survival data for supraglottic cancer. Evaluating HNSCC from different primary sites and with a preponderance of tumors with a high response rate, the irresponsiveness of an underrepresented entity could have been blurred. To our knowledge, our study shows for the first time for the specific entity of supraglottic cancers-although with the given precaution because of the rather low number of cases-the superiority of CRT over RT.

In several reports and reviews such as that of Silver et al., TLM with or without aRT is proposed as the preferred treatment for early supraglottic cancer [32]. Several studies have compared TLM and primary RT. To our knowledge, however, no study to date has proven 
the efficacy of adjuvant RT in supraglottic cancer patients by comparing TLM with and without aRT. There is, however, evidence from Iro et al. and from our present study, that aRT is not sufficiently efficacious in supraglottic cancer patients. While Cooper and Bernier have shown the superiority of aCRT over aRT for HNSCC in general, our data showalthough with a rather small number of patients-that aCRT might be a treatment option superior to aRT for the specific entity of supraglottic cancer patients with positive margins who refuse further surgery and for patients with positive nodal disease $(\mathrm{N}+)$. In the NCCN guidelines, the option to "consider" systemic therapy/RT for supraglottic cancer patients with positive margins is given, while aRT is regarded as the therapy of choice. aCRT with concurrent cisplatin, however, is recommended for locally advanced disease [12]. According to Cooper and Bernier, for HNSCC in general, there is a survival benefit for high risk patients, leading to the recognized recommendation of aCRT in patients having $\mathrm{R}+$ or ECS+. In our data, less than half of the failure patients had a positive resection status (R1) and in none of the patients positive extracapsular spread was reported. Thus, in the entity of supraglottic cancers, patients could experience a survival benefit even if these standard high-risk criteria are not given. Therefore, provided that the patient is fit enough, we suggest chemoradiotherapy for supraglottic cancers in the primary setting at the early stage and in the adjuvant setting for cases at high risk both in the sense of R+ or ECS+ and simply for belonging to this tumor entity with poor prognosis. However, the presumed survival benefit has to be verified in prospective clinical studies.

In accordance with the current guidelines, Ambrosch states that a single-modality therapy with the goal of curing the disease while preserving laryngeal functions is recommended. By applying quality of life (QoL) questionnaires, she convincingly proved that long-term swallow function and voice-related QoL are worse after multimodality treatment [20]. The addition of systemic chemotherapy will further increase the toxicity of adjuvant irradiation. Therefore, our recommendation should be validated in prospective studies with larger numbers of patients.

\section{Materials and Methods}

As reported previously [33], we recruited all index laryngeal cancer patients from the five centers in a well-defined region in southwest Germany that are in charge of the treatment of head and neck cancer patients between 5 January 1998, and 31 December 2004, resulting in a cohort of 810 patients. Patients for this study were identified in two different ways. The first part of the patient cohort was patients who took part in a previous prospective case-control study between 1998 and 2000. For the second part (2001-2004), all patients were identified retrospectively. The charts of all patients were evaluated retrospectively. A follow-up was performed up to March 2015 leading to a total follow-up time of 11 to 17 years. Demographic data and clinical information were obtained from hospital medical records using a standardized form. Vital status as well as date and cause of death were inquired from local registries. Retrospectively, the different therapy groups were checked for comparability with the established confounders sex, age, comorbidities, TNM stage, differentiation, and primary tumor site. OS rates were calculated using the Kaplan-Meier method. Regression analysis was performed using multivariable proportional hazards models. The OS rates of pCRT and pRT, both with the option of salvage total laryngectomy, were compared with those of surgery with adjuvant radiotherapy or adjuvant chemoradiotherapy, as indicated by risk and stage $(\mathrm{OP} \pm \mathrm{a}[\mathrm{C}] \mathrm{RT})$. Survival time was defined as the time from the first diagnosis until death or until 21 March 2015. For the evaluation, patients who moved away from Germany were censored after one month of emigration. $p$-values below 0.05 were regarded as statistically significant. Only overall and disease specific survival estimates are shown. The following variables showed an effect in the univariate analysis $(p<0.20)$ and were included in the multivariable analysis as explanatory variables: age at first diagnosis (continuous), comorbidities, tumor site, TNM status, and therapy modality. Backward selection was used to receive a final model. The proportional hazards assumption was checked by adding a time-dependent version of all the variables in the 
model [34]. The assumption was met for all variables. The M status could not be evaluated as evidence of distant metastases could be proven for only 5 patients. Comorbidity conditions were defined using the Charlson Comorbidity Index (CCI), which summarizes 18 different comorbidities, weighted by severity, in a single score [35]. For this analysis, we used the binary form of the variable, which is set to one for CCI values of two or higher. The data analysis for this paper was generated using SAS/STAT software, Version 14.2 of the SAS System for Windows, copyright (C) 2012 (SAS Institute Inc., Cary, NC, USA). For differentiated evaluation of the efficacy of aRT, criteria for the assessment of success and failure of adjuvant treatment were defined. (See Table 8) Patients were classified as per safety degree of aRT indication (no indication, uncertain, probable, and clear indication) according to $\mathrm{R}$ status and $\mathrm{N}$ stage and categorized as per oncologic outcome in an evaluation matrix.

\section{Conclusions}

In the multi-institutional setting of our observational cohort study, oncological outcome was achieved, which is comparable to that reached in laser centers of excellence. Laser surgery should, however, be performed in academic teaching hospitals or equivalent facilities with sufficient experience and caseload. Adequate care should be exercised in the processing of resected specimens to avoid Rx results. The paramount aim is the clear R0 resection. If free margins are not achieved by TLM, the switch to OPL is recommended.

According to our data and supported by the literature, adjuvant radiotherapy alone is not sufficiently efficacious. There is, however, evidence that chemoradiation is more effective even in supraglottic cancer. Thus, we suggest chemoradiotherapy for supraglottic cancers in the primary setting even at the early stage and in the adjuvant setting for cases at high risk both in the sense of R+ or ECS+ and simply for belonging to this tumor entity with poor prognosis-provided that the patient is fit enough and wishes for maximum safety even at the cost of higher toxicity. However, further prospective studies with larger numbers of patients are warranted.

Author Contributions: Conceptualization, G.D. and H.R.; methodology, H.R.; software, H.R.; validation: G.D., R.W. and E.R.; formal analysis, H.R. and G.D.; resources, P.K.P. and R.W.; data curation, H.R., G.D. and E.R. writing—original draft preparation: G.D.; writing—review and editing, H.R., C.H.-M., R.W., P.K.P. and E.R.; supervision, P.K.P.; project administration: H.R. and C.H.-M. All authors have read and agreed to the published version of the manuscript.

Funding: Data collection of this study was supported by Dietmar Hopp Stiftung GmbH; St. Leon-Rot (grant number: 23011184).

Institutional Review Board Statement: The study was conducted according to the guidelines of the Declaration of Helsinki, and approved by the ethics committee of the Medical University in Heidelberg (Ethics Commission S-141/2008 Medical Faculty).

Informed Consent Statement: The patients who took part in the prospective case-control study (1998-2000) all gave their written informed consent, including long-term follow-up (Ethics Commission 135/97/1997 Medical Faculty). Written informed consent was obtained from the participants through collaborating physicians. For patients who were identified retrospectively (2001-2004), no written consent was required (Ethics Commission S-141/2008 Medical Faculty.)

Data Availability Statement: The datasets generated and analyzed during the current study are available from the corresponding author on reasonable request.

Conflicts of Interest: The authors declare no conflict of interest. The funders had no role in the design of the study; in the collection, analyses, or interpretation of data; in the writing of the manuscript, or in the decision to publish the results. 


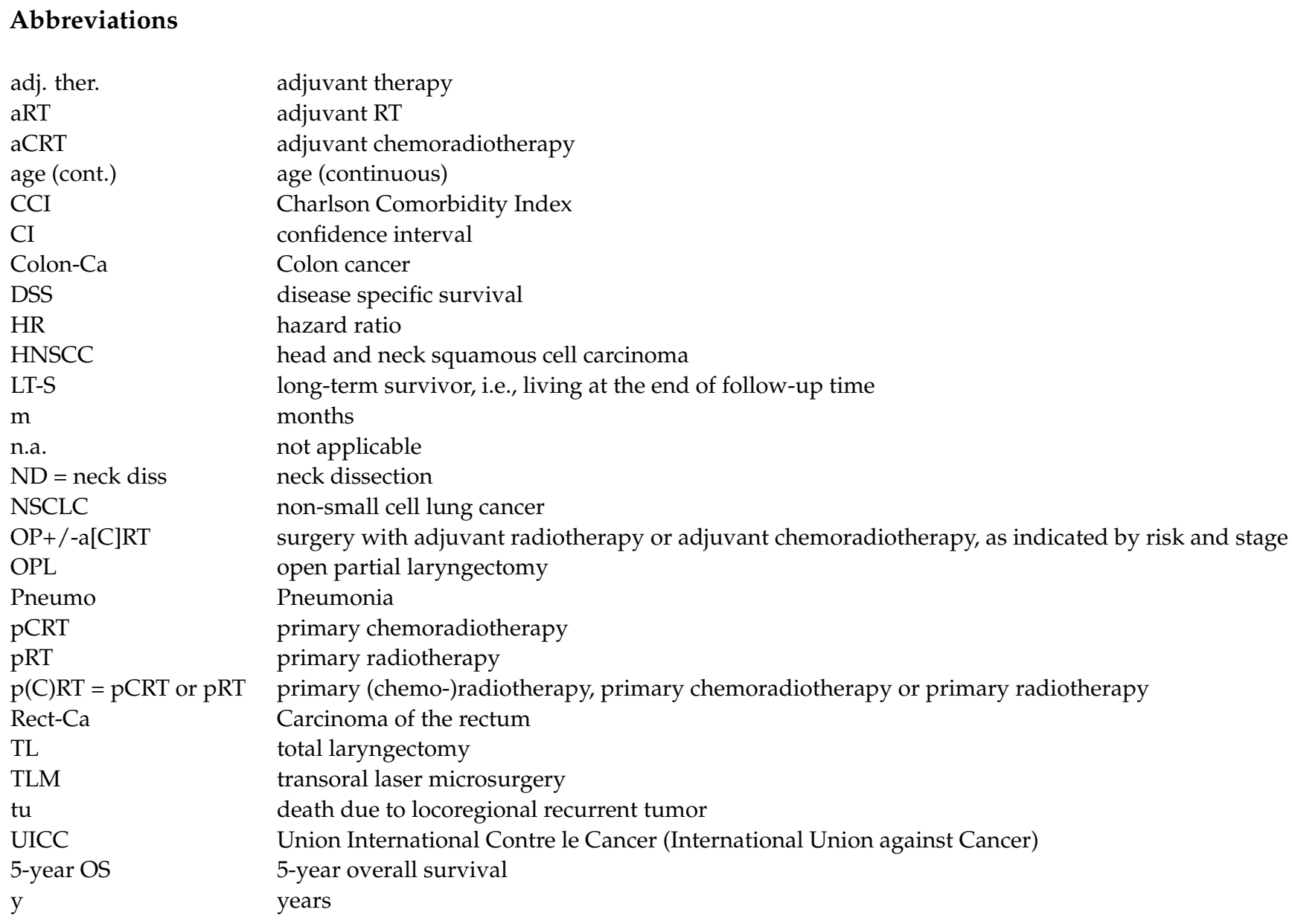

\section{References}

1. Ferlay, J.; Colombet, M.; Soerjomataram, I.; Mathers, C.; Parkin, D.M.; Pineros, M.; Znaor, A.; Bray, F. Estimating the global cancer incidence and mortality in 2018: GLOBOCAN sources and methods. Int. J. Cancer 2019, 144, 1941-1953. [CrossRef] [PubMed]

2. Am Cancer Soc Key Statistics for Laryngeal and Hypopharyngeal Cancers. 2020. Available online: https://www.cancer.org/ content/dam/CRC/PDF/Public/8664.00.pdf (accessed on 16 October 2020).

3. Brandstorp-Boesen, J.; Falk, R.S.; Boysen, M.; Brondbo, K. Long-term trends in gender, T-stage, subsite and treatment for laryngeal cancer at a single center. Eur. Arch. Otorhinolaryngol. 2014, 271, 3233-3239. [CrossRef]

4. Van Dijk, B.A.; Karim-Kos, H.E.; Coebergh, J.W.; Marres, H.A.; de Vries, E. Progress against laryngeal cancer in The Netherlands between 1989 and 2010. Int. J. Cancer 2014, 134, 674-681. [CrossRef] [PubMed]

5. Ramroth, H.; Dietz, A.; Becher, H. Interaction effects and population-attributable risks for smoking and alcohol on laryngeal cancer and its subsites. A case-control study from Germany. Methods Inf. Med. 2004, 43, 499-504. [CrossRef] [PubMed]

6. Talamini, R.; Bosetti, C.; La Vecchia, C.; Dal Maso, L.; Levi, F.; Bidoli, E.; Negri, E.; Pasche, C.; Vaccarella, S.; Barzan, L.; et al Combined effect of tobacco and alcohol on laryngeal cancer risk: A case-control study. Cancer Causes Control CCC 2002, 13, 957-964. [CrossRef]

7. La Vecchia, C.; Zhang, Z.F.; Altieri, A. Alcohol and laryngeal cancer: An update. Eur. J. Cancer Prev. 2008, 17, 116-124. [CrossRef]

8. Cosetti, M.; Yu, G.P.; Schantz, S.P. Five-year survival rates and time trends of laryngeal cancer in the US population. Arch. Otolaryngol. Head Neck Surg. 2008, 134, 370-379. [CrossRef]

9. Patel, K.B.; Nichols, A.C.; Fung, K.; Yoo, J.; MacNeil, S.D. Treatment of early stage Supraglottic squamous cell carcinoma: Meta-analysis comparing primary surgery versus primary radiotherapy. J. Otolaryngol. Head Neck Surg. 2018, 47, 19. [CrossRef]

10. van der Woerd, B.; Patel, K.B.; Nichols, A.C.; Fung, K.; Yoo, J.; MacNeil, S.D. Functional outcomes in early (T1/T2) supraglottic cancer: A systematic review. J. Otolaryngol. Head Neck Surg. 2018, 47, 76. [CrossRef]

11. Raitiola, H.; Pukander, J.; Laippala, P. Glottic and supraglottic laryngeal carcinoma: Differences in epidemiology, clinical characteristics and prognosis. Acta Otolaryngol. 1999, 119, 847-851. [CrossRef]

12. Pfister, D.G. NCCN Clinical Practice Guidelines in Oncology in Head and Neck Cancers (Version 2.2020). Available online: https:/ / www.nccn.org/professionals/physician_gls/pdf/head-and-neck.pdf (accessed on 6 December 2020).

13. Silvestri, F.; Bussani, R.; Stanta, G.; Cosatti, C.; Ferlito, A. Supraglottic versus glottic laryngeal cancer: Epidemiological and pathological aspects. Orl J. Oto-Rhino-Laryngol. Its Relat. Spec. 1992, 54, 43-48. [CrossRef] [PubMed] 
14. Iro, H.; Mantsopoulos, K.; Zenk, J.; Waldfahrer, F.; Psychogios, G. Results of transoral laser resection in T1-2 oropharyngeal, hypopharyngeal and laryngeal carcinomas. Laryngo-Rhino-Otologie 2011, 90, 481-485. [CrossRef] [PubMed]

15. Vaughan, C.W. Transoral laryngeal surgery using the $\mathrm{CO}_{2}$ laser: Laboratory experiments and clinical experience. Laryngoscope 1978, 88, 1399-1420. [CrossRef] [PubMed]

16. Davis, R.K.; Shapshay, S.M.; Strong, M.S.; Hyams, V.J. Transoral partial supraglottic resection using the $\mathrm{CO}_{2}$ laser. Laryngoscope 1983, 93, 429-432. [CrossRef]

17. Ambrosch, P.; Kron, M.; Steiner, W. Carbon dioxide laser microsurgery for early supraglottic carcinoma. Ann. Otol. Rhinol. Laryngol. 1998, 107, 680-688. [CrossRef] [PubMed]

18. Iro, H.; Waldfahrer, F.; Altendorf-Hofmann, A.; Weidenbecher, M.; Sauer, R.; Steiner, W. Transoral laser surgery of supraglottic cancer: Follow-up of 141 patients. Arch. Otolaryngol. Head Neck Surg. 1998, 124, 1245-1250. [CrossRef]

19. Rodrigo, J.P.; Suarez, C.; Silver, C.E.; Rinaldo, A.; Ambrosch, P.; Fagan, J.J.; Genden, E.M.; Ferlito, A. Transoral laser surgery for supraglottic cancer. Head Neck 2008, 30, 658-666. [CrossRef]

20. Ambrosch, P.; Gonzalez-Donate, M.; Fazel, A.; Schmalz, C.; Hedderich, J. Transoral Laser Microsurgery for Supraglottic Cancer. Front. Oncol. 2018, 8, 158. [CrossRef]

21. Davis, R.K.; Kriskovich, M.D.; Galloway, E.B., 3rd; Buntin, C.S.; Jepsen, M.C. Endoscopic supraglottic laryngectomy with postoperative irradiation. Ann. Otol. Rhinol. Laryngol. 2004, 113, 132-138. [CrossRef]

22. Steiner, W.; Ambrosch, P. Stellenwert der Laserchirurgie bei Tumoren der oberen Luft-und Speisewege. Der. Onkol. 1996, 2 , 346-351. [CrossRef]

23. Rudert, H.H.; Werner, J.A.; Hoft, S. Transoral carbon dioxide laser resection of supraglottic carcinoma. Ann. Otol. Rhinol. Laryngol. 1999, 108, 819-827. [CrossRef] [PubMed]

24. Ambrosch, P.; Brinck, U.; Fischer, G.; Steiner, W. Special aspects of histopathologic diagnosis in laser microsurgery of cancers of the upper aerodigestive tract. Laryngo-Rhino-Otologie 1994, 73, 78-83. [CrossRef] [PubMed]

25. Jumaily, M.; Faraji, F.; Osazuwa-Peters, N.; Walker, R.J.; Ward, G.M. Prognostic significance of surgical margins after transoral laser microsurgery for early-stage glottic squamous cell carcinoma. Oral Oncol. 2019, 97, 105-111. [CrossRef] [PubMed]

26. Hanna, J.; Brauer, P.R.; Morse, E.; Mehra, S. Margins in Laryngeal Squamous Cell Carcinoma Treated with Transoral Laser Microsurgery: A National Database Study. Otolaryngol. Head Neck Surg. 2019, 161, 986-992. [CrossRef]

27. Pedregal-Mallo, D.; Sanchez Canteli, M.; Lopez, F.; Alvarez-Marcos, C.; Llorente, J.L.; Rodrigo, J.P. Oncological and functional outcomes of transoral laser surgery for laryngeal carcinoma. Eur. Arch. Otorhinolaryngol. 2018, 275, 2071-2077. [CrossRef]

28. Fiz, I.; Mazzola, F.; Fiz, F.; Marchi, F.; Filauro, M.; Paderno, A.; Parrinello, G.; Piazza, C.; Peretti, G. Impact of Close and Positive Margins in Transoral Laser Microsurgery for Tis-T2 Glottic Cancer. Front. Oncol. 2017, 7, 245. [CrossRef]

29. Harreus, U. Chirurgische Onkologie, Halschirurgie. In HNO Update 2020; Iro, H., Verse, T., Wollenberg, B., Zenk, J., Eds.; Med Update: Mainz, Germany, 2020.

30. Cooper, J.S.; Pajak, T.F.; Forastiere, A.A.; Jacobs, J.; Campbell, B.H.; Saxman, S.B.; Kish, J.A.; Kim, H.E.; Cmelak, A.J.; Rotman, M.; et al. Postoperative concurrent radiotherapy and chemotherapy for high-risk squamous-cell carcinoma of the head and neck. $N$. Engl. J. Med. 2004, 350, 1937-1944. [CrossRef]

31. Bernier, J.; Domenge, C.; Ozsahin, M.; Matuszewska, K.; Lefebvre, J.L.; Greiner, R.H.; Giralt, J.; Maingon, P.; Rolland, F.; Bolla, M.; et al. Postoperative irradiation with or without concomitant chemotherapy for locally advanced head and neck cancer. N. Engl. J. Med. 2004, 350, 1945-1952. [CrossRef]

32. Silver, C.E.; Beitler, J.J.; Shaha, A.R.; Rinaldo, A.; Ferlito, A. Current trends in initial management of laryngeal cancer: The declining use of open surgery. Eur. Arch. Otorhinolaryngol. 2009, 266, 1333-1352. [CrossRef]

33. Dyckhoff, G.; Plinkert, P.K.; Ramroth, H. A change in the study evaluation paradigm reveals that larynx preservation compromises survival in T4 laryngeal cancer patients. BMC Cancer 2017, 17, 609. [CrossRef]

34. Allison, P. Survival Analysis Using SAS A Practical Guide, 2nd ed.; SAS Institute Inc.: Cary, NC, USA, 2010.

35. Charlson, M.E.; Pompei, P.; Ales, K.L.; MacKenzie, C.R. A new method of classifying prognostic comorbidity in longitudinal studies: Development and validation. J. Chronic Dis. 1987, 40, 373-383. [CrossRef] 